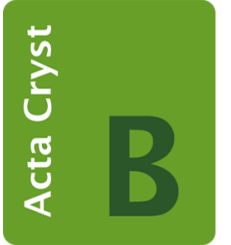

STRUCTURAL SCIENCE

CRYSTAL ENGINEERING

MATERIALS

Volume 77 (2021)

Supporting information for article:

High-pressure and environment effects in selenourea and its labile crystal field around molecules

Kinga Roszak and Andrzej Katrusiak 
S1. Tables

S1.1. Crystallographic details 
Table S1 Crystallographic data of selenourea phase $\alpha$ and experimental details.

\begin{tabular}{|c|c|c|c|c|}
\hline Pressure $(\mathrm{GPa})$ & 0.0001 & 0.0001 & 0.10 & 0.18 \\
\hline CCDC number & 2061316 & 2061317 & 2061318 & 2061319 \\
\hline Formula & $\mathrm{SeC}\left(\mathrm{NH}_{2}\right)_{2}$ & $\mathrm{SeC}\left(\mathrm{NH}_{2}\right)_{2}$ & $\mathrm{SeC}\left(\mathrm{NH}_{2}\right)_{2}$ & $\mathrm{SeC}\left(\mathrm{NH}_{2}\right)_{2}$ \\
\hline Crystal form & Polymorph $\alpha$ & Polymorph $\alpha$ & Polymorph $\alpha$ & Polymorph $\alpha$ \\
\hline Formula weight & 123.02 & 123.02 & 123.02 & 123.02 \\
\hline Wavelength $(\AA)$ & 0.71073 & 0.71073 & 0.71073 & 0.71073 \\
\hline Crystal system & trigonal & trigonal & trigonal & trigonal \\
\hline Space group & $P 3_{2}$ & $P 3_{2}$ & $P 3_{2}$ & $P 3_{2}$ \\
\hline $\begin{array}{ll}\text { Unit cell dimensions }(\AA): & a \\
& c\end{array}$ & $\begin{array}{l}15.2768(3) \\
13.0239(3)\end{array}$ & $\begin{array}{l}15.2792(15) \\
13.0326(9)\end{array}$ & $\begin{array}{l}15.2661(14) \\
13.0260(8)\end{array}$ & $\begin{array}{l}15.3025(10) \\
13.0498(7)\end{array}$ \\
\hline Volume $\left(\AA^{3}\right)$ & $2632.31(12)$ & $2634.9(5)$ & $2629.0(5)$ & $2646.4(4)$ \\
\hline $\mathrm{Z} / \mathrm{Z}^{\prime}$ & $27 / 9$ & $27 / 9$ & $27 / 9$ & $27 / 9$ \\
\hline Density $\left(\mathrm{g} / \mathrm{cm}^{3}\right)$ & 2.095 & 2.093 & 2.098 & 2.084 \\
\hline Absorption coeff. $\left(\mathrm{mm}^{-1}\right)$ & 9.399 & 9.390 & 9.411 & 9.349 \\
\hline Radiation type, $\lambda(\AA)$ & $\operatorname{MoK} \alpha, 0.71073$ & MoK $\alpha, 0.71073$ & $\operatorname{MoK} \alpha, 0.71073$ & $\operatorname{MoK} \alpha, 0.71073$ \\
\hline $\mathrm{F}(000)$ & 1566 & 1566 & 1566 & 1566 \\
\hline Crystal size (mm) & $0.30 \cdot 0.10 \cdot 0.05$ & $0.30 \cdot 0.25 \cdot 0.05$ & $0.30 \cdot 0.25 \cdot 0.05$ & $0.48 \cdot 0.10 \cdot 0.05$ \\
\hline$\Theta$-range for data $\left(^{\circ}\right)$ & 6.158 to 54.188 & 8.73 to 54.046 & 8.736 to 54.084 & 8.802 to 50.000 \\
\hline Min/max indices $h, k, 1$ & $\begin{array}{l}-19 / 19,-18 / 19, \\
-16 / 15\end{array}$ & $\begin{array}{l}-13 / 13,-19 / 19, \\
-16 / 16\end{array}$ & $\begin{array}{l}-13 / 13,-19 / 19, \\
-16 / 16\end{array}$ & $\begin{array}{l}\text { 11/-11, 18/-18, } \\
15 /-15\end{array}$ \\
\hline Reflect. collected/unique & $37197 / 7250$ & $13716 / 4059$ & $13651 / 4062$ & $10411 / 2981$ \\
\hline $\mathrm{R}_{\text {int }}$ & 0.0630 & 0.0969 & 0.0900 & 0.0875 \\
\hline Data/restrains/parameters & $7250 / 0 / 326$ & $4059 / 133 / 326$ & $4062 / 137 / 326$ & $2981 / 97 / 326$ \\
\hline Goodness-of-fit on $\mathrm{F}^{2}$ & 1.085 & 1.020 & 1.020 & 1.013 \\
\hline Final $R_{1} / w R_{2}\left(\mathrm{I}>2 \sigma_{1}\right)$ & $0.0499 / 0.1049$ & $0.0962 / 0.1721$ & $0.0984 / 0.1351$ & $0.0684 / 0.1545$ \\
\hline$R_{1} / w R_{2}$ (all data) & $0.0830 / 0.1189$ & $0.1225 / 0.1971$ & $0.1087 / 0.1878$ & $0.1271 / 0.1876$ \\
\hline
\end{tabular}


Table S1 (continued). Crystallographic data of selenourea phase $\alpha$ and experimental details.

\begin{tabular}{|c|c|c|}
\hline Pressure $(\mathrm{GPa})$ & 0.20 & 0.23 \\
\hline CCDC number & 2061320 & 2061315 \\
\hline Formula & $\mathrm{SeC}\left(\mathrm{NH}_{2}\right)_{2}$ & $\mathrm{SeC}\left(\mathrm{NH}_{2}\right)_{2}$ \\
\hline Crystal form & Polymorph $\alpha$ & Polymorph $\alpha$ \\
\hline Formula weight & 123.02 & 123.02 \\
\hline Wavelength $(\AA)$ & 0.71073 & 0.71073 \\
\hline Crystal system & trigonal & trigonal \\
\hline Space group & $P 3_{2}$ & $P 3_{2}$ \\
\hline 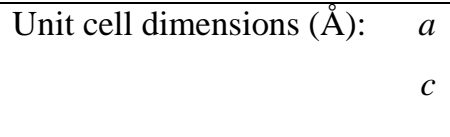 & $\begin{array}{l}15.1445(10) \\
12.9631(7)\end{array}$ & $\begin{array}{l}15.243(4) \\
13.009(3)\end{array}$ \\
\hline Volume $\left(\AA^{3}\right)$ & $2574.8(4)$ & $2617.7(15)$ \\
\hline $\mathrm{Z} / \mathrm{Z}^{\prime}$ & $27 / 9$ & $27 / 9$ \\
\hline Density $\left(\mathrm{g} / \mathrm{cm}^{3}\right)$ & 2.142 & 2.107 \\
\hline Absorption coeff. $\left(\mathrm{mm}^{-1}\right)$ & 9.609 & 9.451 \\
\hline Radiation type, $\lambda(\AA)$ & $\operatorname{MoK} \alpha, 0.71073$ & $\operatorname{MoK} \alpha, 0.71073$ \\
\hline $\mathrm{F}(000)$ & 1566 & 1566 \\
\hline Crystal size (mm) & $0.35 \cdot 0.20 \cdot 0.05$ & $0.30 \cdot 0.25 \cdot 0.05$ \\
\hline$\Theta$-range for data $\left(^{\circ}\right)$ & 8.804 to 53.406 & 9.264 to 52.45 \\
\hline Min/max indices $\mathrm{h}, \mathrm{k}, \mathrm{l}$ & $\begin{array}{l}-11 / 11,-19 / 18, \\
-16 / 16\end{array}$ & $\begin{array}{l}-5 / 8,-13 / 12, \\
-16 / 14\end{array}$ \\
\hline Reflect. collected/unique & $10694 / 3131$ & $2691 / 1795$ \\
\hline $\mathrm{R}_{\text {int }}$ & 0.0889 & 0.0563 \\
\hline Data/restrains/parameters & $3131 / 103 / 326$ & $1795 / 179 / 326$ \\
\hline Goodness-of-fit on $\mathrm{F}^{2}$ & 1.035 & 1.033 \\
\hline Final $R_{1} / w R_{2}\left(\mathrm{I}>2 \sigma_{1}\right)$ & $0.0717 / 0.1620$ & $0.0823 / 0.1874$ \\
\hline$R_{1} / w R_{2}$ (all data) & $0.1364 / 0.2050$ & $0.1568 / 0.2366$ \\
\hline
\end{tabular}


Table S2. Crystallographic data of selenourea phase $\beta$ and experimental details.

\begin{tabular}{|c|c|c|c|c|}
\hline Pressure $(\mathrm{GPa})$ & 0.21 & 0.28 & 0.38 & 0.58 \\
\hline CCDC number & 2061307 & 2061308 & 2061309 & 2061310 \\
\hline Formula & $\mathrm{SeC}\left(\mathrm{NH}_{2}\right)_{2}$ & $\mathrm{SeC}\left(\mathrm{NH}_{2}\right)_{2}$ & $\mathrm{SeC}\left(\mathrm{NH}_{2}\right)_{2}$ & $\mathrm{SeC}\left(\mathrm{NH}_{2}\right)_{2}$ \\
\hline Crystal form & Polymorph $\beta$ & Polymorph $\beta$ & Polymorph $\beta$ & Polymorph $\beta$ \\
\hline Formula weight & 123.02 & 123.02 & 123.02 & 123.02 \\
\hline Wavelength $(\AA)$ & 0.71073 & 0.71073 & 0.71073 & 0.71073 \\
\hline Crystal system & monoclinic & monoclinic & monoclinic & monoclinic \\
\hline Space group & $P 2_{1} / c$ & $P 2_{1} / c$ & $P 2_{1} / c$ & $P 2_{1} / c$ \\
\hline 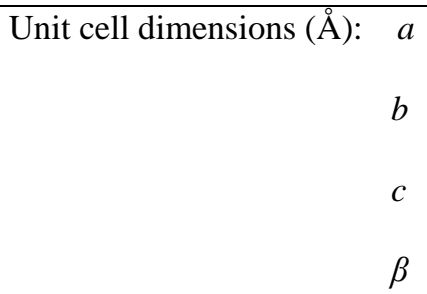 & $\begin{array}{l}7.6155(13) \\
8.4109(7) \\
11.709(3) \\
100.718(16)\end{array}$ & $\begin{array}{l}7.6122(13) \\
8.4048(7) \\
11.707(3) \\
100.702(16)\end{array}$ & $\begin{array}{l}7.5985(3) \\
8.3869(7) \\
11.684(2) \\
100.609(9)\end{array}$ & $\begin{array}{l}7.570(3) \\
8.3144(14) \\
11.604(4) \\
100.75(3)\end{array}$ \\
\hline Volume $\left(\AA^{3}\right)$ & $736.9(2)$ & $736.0(2)$ & $731.87(14)$ & $717.6(4)$ \\
\hline $\mathrm{Z} / \mathrm{Z}^{\prime}$ & $8 / 2$ & $8 / 2$ & $8 / 2$ & $8 / 2$ \\
\hline Density $\left(\mathrm{g} / \mathrm{cm}^{3}\right)$ & 2.218 & 2.220 & 2.233 & 2.278 \\
\hline Absorption coeff. $\left(\mathrm{mm}^{-1}\right)$ & 9.948 & 9.960 & 10.016 & 10.216 \\
\hline Radiation type, $\lambda(\AA)$ & $\operatorname{MoK} \alpha, 0.71073$ & $\mathrm{MoK} \alpha, 0.71073$ & $\operatorname{MoK} \alpha, 0.71073$ & $\operatorname{MoK} \alpha, 0.71073$ \\
\hline $\bar{F}(000)$ & 464 & 464 & 464 & 464 \\
\hline Crystal size $(\mathrm{mm})$ & $0.40 \cdot 0.30 \cdot 0.05$ & $0.40 \cdot 0.30 \cdot 0.05$ & $0.40 \cdot 0.30 \cdot 0.05$ & $0.40 \cdot 0.30 \cdot 0.05$ \\
\hline$\Theta$-range for data $\left(^{\circ}\right)$ & 8.538 to 53.538 & 8.54 to 53.99 & 8.552 to 54.084 & 8.612 to 54.048 \\
\hline Min/max indices $\mathrm{h}, \mathrm{k}, \mathrm{l}$ & $-7 / 7,-10 / 10,-9 / 9$ & $-7 / 7,-10 / 10,-9 / 9$ & $-9 / 9,-9 / 9,-8 / 8$ & $-7 / 7,-10 / 10,-9 / 9$ \\
\hline Reflect. collected/unique & $3768 / 570$ & $3774 / 571$ & $3681 / 616$ & $3394 / 556$ \\
\hline $\mathrm{R}_{\text {int }}$ & 0.0318 & 0.0312 & 0.0281 & 0.0488 \\
\hline Data/restrains/parameters & $570 / 0 / 74$ & $571 / 0 / 74$ & $616 / 0 / 74$ & $556 / 6 / 73$ \\
\hline Goodness-of-fit on $\mathrm{F}^{2}$ & 1.112 & 1.149 & 1.184 & 1.062 \\
\hline Final $R_{1} / w R_{2}\left(\mathrm{I}>2 \sigma_{1}\right)$ & $0.0240 / 0.0516$ & $0.0222 / 0.0490$ & $0.0241 / 0.0592$ & $0.0356 / 0.0747$ \\
\hline$R_{1} / w R_{2}$ (all data) & $0.0327 / 0.0541$ & $0.0294 / 0.0520$ & $0.0305 / 0.0617$ & $0.0517 / 0.0826$ \\
\hline
\end{tabular}


Table S2 (continued). Crystallographic data of selenourea phase $\beta$ and experimental details.

\begin{tabular}{|c|c|c|c|c|}
\hline Pressure $(\mathrm{GPa})$ & 1.01 & 1.79 & 2.52 & 3.20 \\
\hline CCDC number & 2061311 & 2061312 & 2061313 & 2061314 \\
\hline Formula & $\mathrm{SeC}\left(\mathrm{NH}_{2}\right)_{2}$ & $\mathrm{SeC}\left(\mathrm{NH}_{2}\right)_{2}$ & $\mathrm{SeC}\left(\mathrm{NH}_{2}\right)_{2}$ & $\mathrm{SeC}\left(\mathrm{NH}_{2}\right)_{2}$ \\
\hline Crystal form & Polymorph $\beta$ & Polymorph $\beta$ & Polymorph $\beta$ & Polymorph $\beta$ \\
\hline Formula weight & 123.02 & 123.02 & 123.02 & 123.02 \\
\hline Wavelength $(\AA)$ & 0.71073 & 0.71073 & 0.71073 & 0.71073 \\
\hline Crystal system & monoclinic & monoclinic & monoclinic & monoclinic \\
\hline Space group & $P 2_{1} / c$ & $P 2_{1} / c$ & $P 2_{1} / c$ & $P 2_{1} / c$ \\
\hline 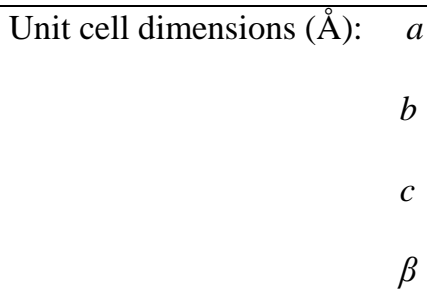 & $\begin{array}{l}7.444(3) \\
8.231(8) \\
11.362(3) \\
100.46(3)\end{array}$ & $\begin{array}{l}7.4559(11) \\
8.117(6) \\
11.373(11) \\
100.53(3)\end{array}$ & $\begin{array}{l}7.3826(5) \\
8.0113(5) \\
11.135(5) \\
100.419(16)\end{array}$ & $\begin{array}{l}7.301(6) \\
7.909(5) \\
11.066(6) \\
102.60(1)\end{array}$ \\
\hline Volume $\left(\AA^{3}\right)$ & $684.7(15)$ & $676.7(8)$ & $647.7(3)$ & $623.6(7)$ \\
\hline $\bar{Z} / \mathrm{Z}^{\prime}$ & $8 / 2$ & $8 / 2$ & $8 / 2$ & $8 / 2$ \\
\hline Density $\left(\mathrm{g} / \mathrm{cm}^{3}\right)$ & 2.387 & 2.415 & 2.523 & 2.621 \\
\hline Absorption coeff. $\left(\mathrm{mm}^{-1}\right)$ & 10.707 & 10.833 & 11.318 & 11.755 \\
\hline Radiation type, $\lambda(\AA)$ & $\operatorname{MoK} \alpha, 0.71073$ & $\operatorname{MoK} \alpha, 0.71073$ & $\operatorname{MoK} \alpha, 0.71073$ & $\operatorname{MoK} \alpha, 0.71073$ \\
\hline $\bar{F}(000)$ & 464 & 464 & 464 & 464 \\
\hline Crystal size $(\mathrm{mm})$ & $0.30 \cdot 0.25 \cdot 0.05$ & $0.20 \cdot 0.10 \cdot 0.05$ & $0.20 \cdot 0.10 \cdot 0.05$ & $0.20 \cdot 0.10 \cdot 0.05$ \\
\hline$\Theta$-range for data $\left(^{\circ}\right)$ & 9.952 to 51.526 & 11.128 to 53.344 & 10.18 to 53.282 & 8.762 to 50.964 \\
\hline Min/max indices $\mathrm{h}, \mathrm{k}, \mathrm{l}$ & $-7 / 7,-3 / 3,-13 / 13$ & $-9 / 9,-4 / 4,-5 / 5$ & $-9 / 9,-10 / 10,-4 / 5$ & $-7 / 7,-7 / 7,-4 / 4$ \\
\hline Reflect. collected/unique & $638 / 178$ & $531 / 141$ & $2838 / 320$ & $458 / 175$ \\
\hline $\mathrm{R}_{\text {int }}$ & 0.2368 & 0.0912 & 0.0676 & 0.1169 \\
\hline Data/restrains/parameters & $178 / 43 / 74$ & $141 / 76 / 74$ & $320 / 65 / 74$ & $175 / 52 / 73$ \\
\hline Goodness-of-fit on $\mathrm{F}^{2}$ & 0.953 & 1.189 & 1.078 & 1.020 \\
\hline Final $R_{1} / w R_{2}\left(\mathrm{I}>2 \sigma_{1}\right)$ & $0.0458 / 0.0464$ & $0.0350 / 0.0739$ & $0.0460 / 0.1033$ & $0.0616 / 0.1427$ \\
\hline$R_{1} / w R_{2}$ (all data) & $0.0750 / 0.0568$ & $0.0716 / 0.0933$ & $0.0682 / 0.1194$ & $0.1101 / 0.1891$ \\
\hline
\end{tabular}


Table S3. Crystallographic data of selenourea duotrito hydrate and experimental details.

\begin{tabular}{|c|c|c|c|}
\hline Pressure $(\mathrm{GPa})$ & 0.52 & 0.80 & 1.10 \\
\hline CCDC number & 2061304 & 2061305 & 2061306 \\
\hline Formula & $3 \mathrm{SeC}\left(\mathrm{NH}_{2}\right)_{2} \cdot \mathrm{H}_{2} \mathrm{O}$ & $3 \mathrm{SeC}\left(\mathrm{NH}_{2}\right)_{2} \cdot \mathrm{H}_{2} \mathrm{O}$ & $3 \mathrm{SeC}\left(\mathrm{NH}_{2}\right)_{2} \cdot \mathrm{H}_{2} \mathrm{O}$ \\
\hline Crystal form & duotritohydrate & duotritohydrate & duotritohydrate \\
\hline Formula weight & 405.10 & 405.10 & 405.10 \\
\hline Wavelength $(\AA)$ & 0.71073 & 0.71073 & 0.71073 \\
\hline Crystal system & monoclinic & monoclinic & monoclinic \\
\hline Space group & $C 2 / c$ & $C 2 / c$ & $C 2 / c$ \\
\hline 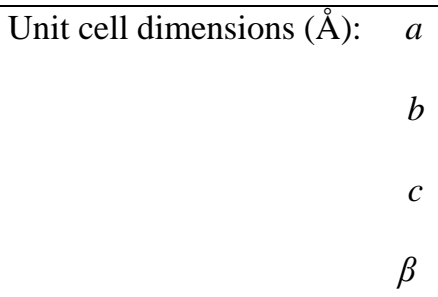 & $\begin{array}{l}8.2698(12) \\
8.8726(8) \\
16.913(3) \\
90.205(17)\end{array}$ & $\begin{array}{l}8.238(2) \\
8.8548(4) \\
16.8134(11) \\
90.628(14)\end{array}$ & $\begin{array}{l}8.1817(5) \\
8.8186(10) \\
16.7510(10) \\
90.979(6)\end{array}$ \\
\hline Volume $\left(\AA^{3}\right)$ & $1241.0(3)$ & $1226.4(3)$ & $1208.43(17)$ \\
\hline $\mathrm{Z} / \mathrm{Z}^{\prime}$ & $4 / 0.5$ & $4 / 0.5$ & $4 / 0.5$ \\
\hline Density $\left(\mathrm{g} / \mathrm{cm}^{3}\right)$ & 2.168 & 2.194 & 2.227 \\
\hline Absorption coeff. $\left(\mathrm{mm}^{-1}\right)$ & 8.883 & 8.988 & 9.122 \\
\hline Radiation type, $\lambda(\AA)$ & $\operatorname{MoK} \alpha, 0.71073$ & $\operatorname{MoK} \alpha, 0.71073$ & $\operatorname{MoK} \alpha, 0.71073$ \\
\hline $\mathrm{F}(000)$ & 776 & 776 & 776 \\
\hline Crystal size $(\mathrm{mm})$ & $0.25 \cdot 0.10 \cdot 0.05$ & $0.25 \cdot 0.20 \cdot 0.05$ & $0.35 \cdot 0.20 \cdot 0.05$ \\
\hline$\Theta$-range for data $\left(^{\circ}\right)$ & 6.736 to 52.728 & 9.208 to 53.36 & 9.916 to 53.744 \\
\hline Min/max indices $\mathrm{h}, \mathrm{k}, \mathrm{l}$ & $-9 / 9,-10 / 10,-16 / 16$ & $-5 / 5,-10 / 10,-19 / 20$ & $-9 / 9,-7 / 8,-19 / 19$ \\
\hline Reflect. collected/unique & $3221 / 584$ & $3105 / 473$ & $3029 / 520$ \\
\hline $\mathrm{R}_{\text {int }}$ & 0.1610 & 0.0309 & 0.0334 \\
\hline Data/restrains/parameters & $584 / 0 / 69$ & $473 / 0 / 69$ & $520 / 0 / 69$ \\
\hline Goodness-of-fit on $\mathrm{F}^{2}$ & 0.968 & 1.154 & 1.066 \\
\hline Final $R_{1} / w R_{2}\left(\mathrm{I}>2 \sigma_{1}\right)$ & $0.0477 / 0.1022$ & $0.0225 / 0.0539$ & $0.0230 / 0.0509$ \\
\hline$R_{1} / w R_{2}$ (all data) & $0.0734 / 0.1165$ & $0.0247 / 0.0549$ & $0.0286 / 0.0528$ \\
\hline
\end{tabular}




\section{S2. Figures}

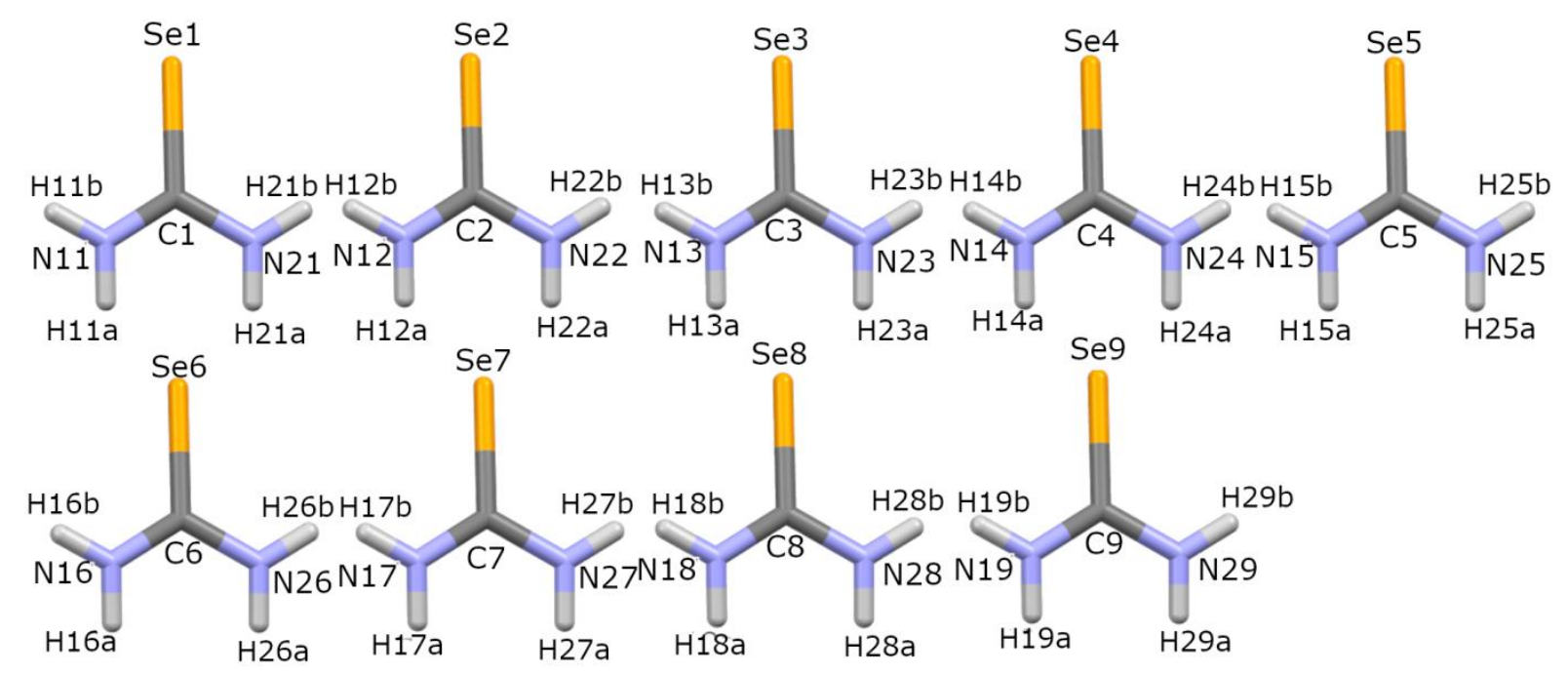

Figure S1 Atomic labels of symmetry-independent molecules in Selenourea phase $\alpha$.

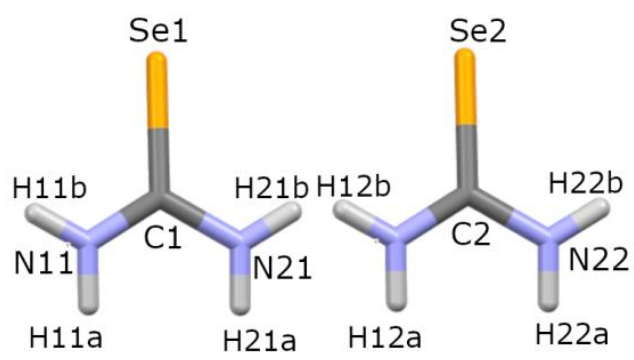

Figure S2 Atomic labels of symmetry-independent molecules in Selenourea phase $\beta$.

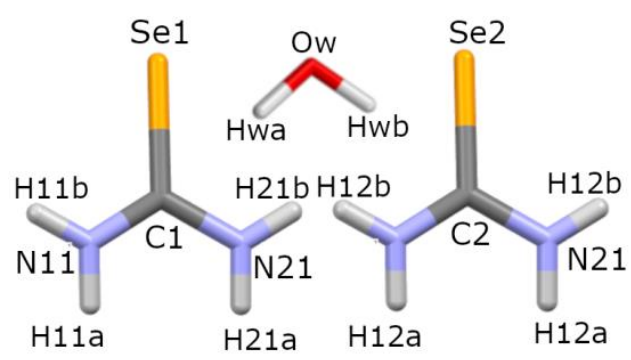

Figure S3 Atomic labels of symmetry-independent molecules in Selenourea duotritohydrate. 


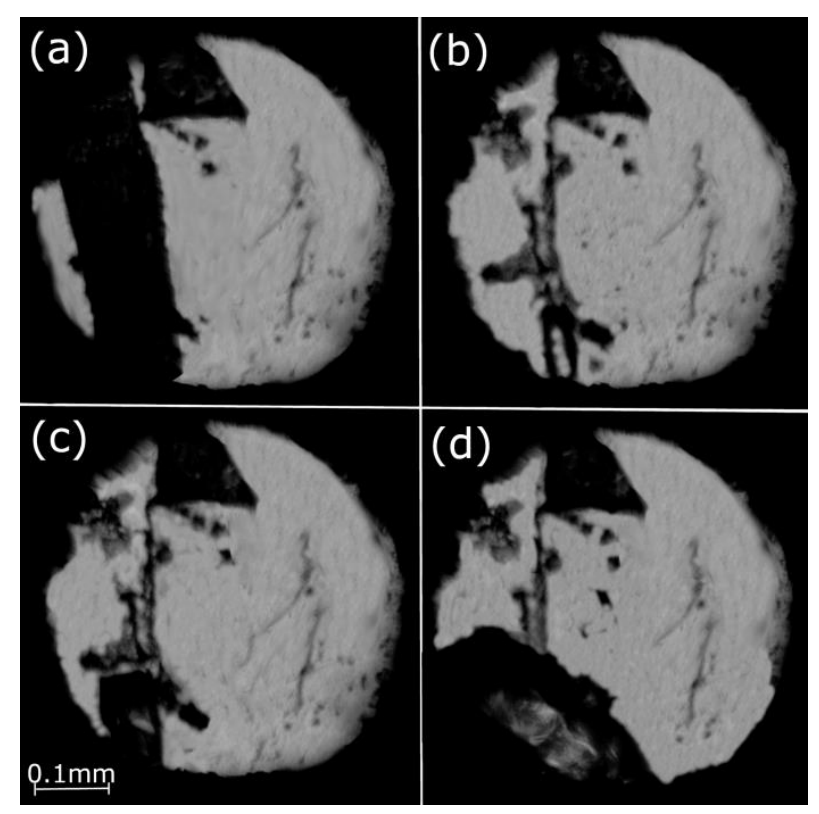

Figure S4 Stages of $3 \mathrm{SeC}\left(\mathrm{NH}_{2}\right)_{2} \cdot 2 \mathrm{H}_{2} \mathrm{O}$ single-crystal isochoric growth from the aqueous solution at: (a) $345 \mathrm{~K}$, (b) $322 \mathrm{~K}$, (c) $318 \mathrm{~K}$ and (d) $0.52 \mathrm{GPa} / 296 \mathrm{~K}$. The ruby chip for pressure calibration is located close to the gasket upper edge.

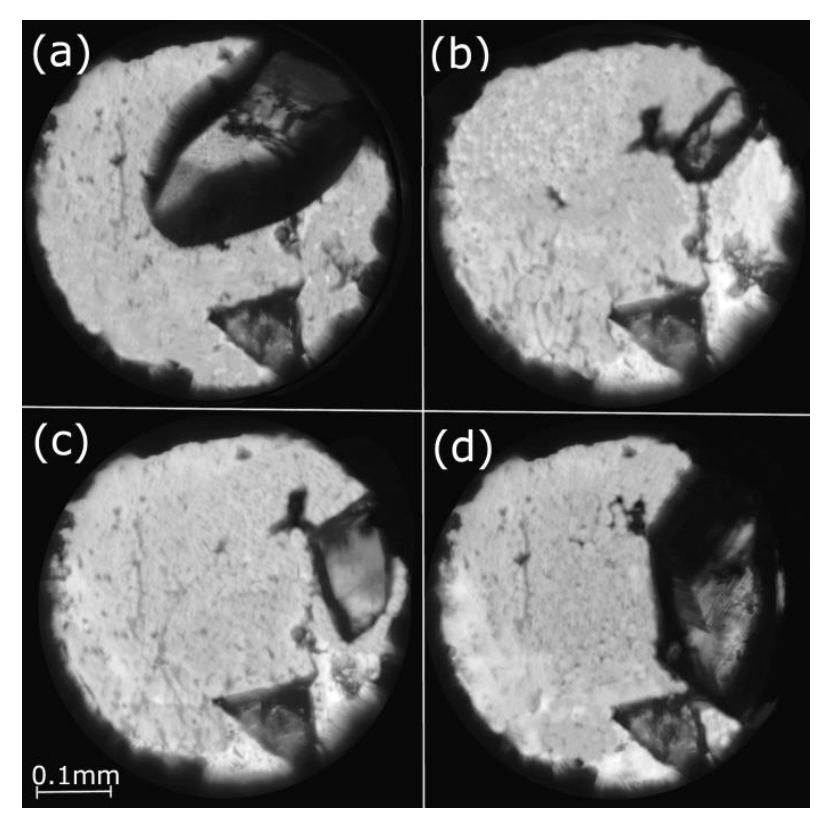

Figure 55 Stages of $3 \mathrm{SeC}\left(\mathrm{NH}_{2}\right)_{2} \cdot 2 \mathrm{H}_{2} \mathrm{O}$ single-crystal isochoric growth from the aqueous solution at: (a) $393 \mathrm{~K}$, (b) $373 \mathrm{~K}$, (c) $323 \mathrm{~K}$ and (d) $1.10 \mathrm{GPa} / 296 \mathrm{~K}$. The ruby chip for pressure calibration is located close to the gasket upper edge. 


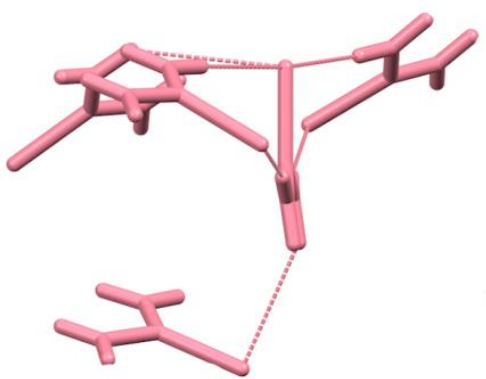

molecule 1
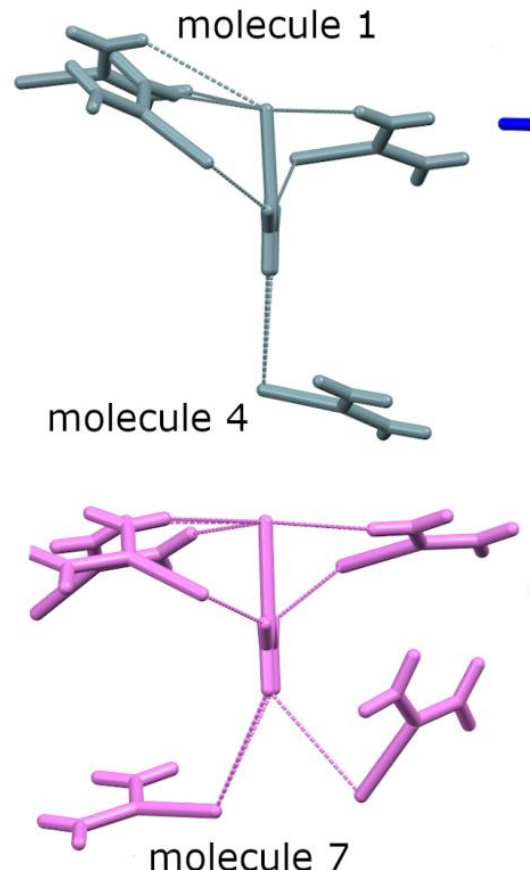
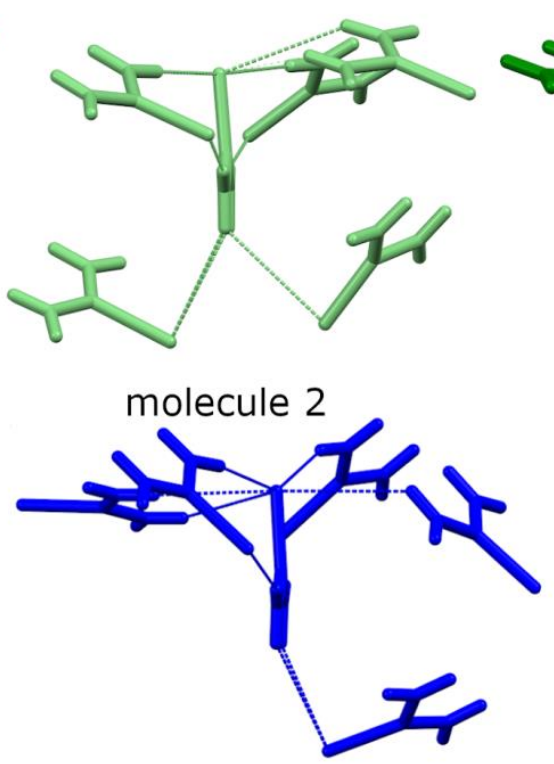

molecule 5

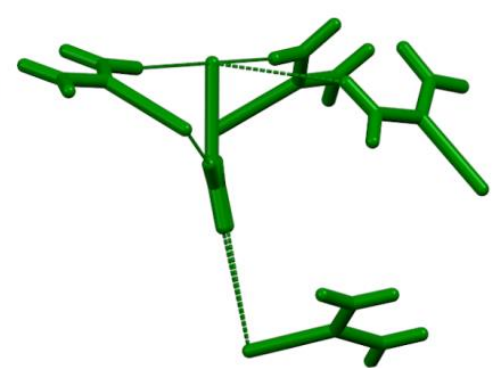

molecule 3

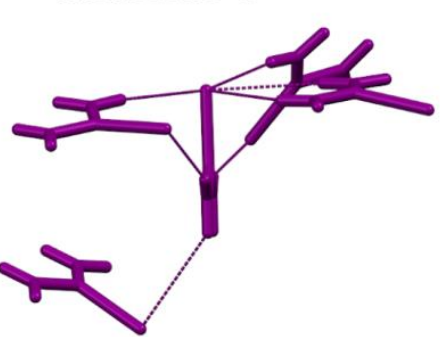

molecule 6

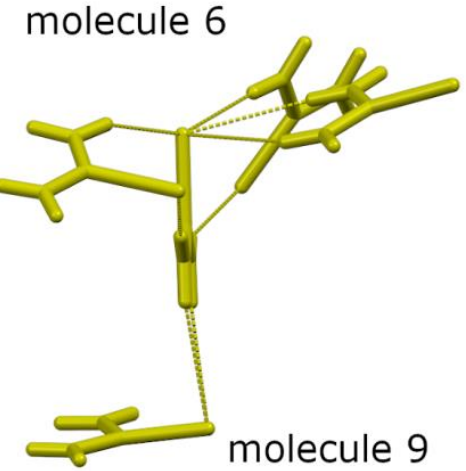

Figure S6 The components of Figure 6a shown for each of the central molecules viewed in the direction parallel to its average plane. 

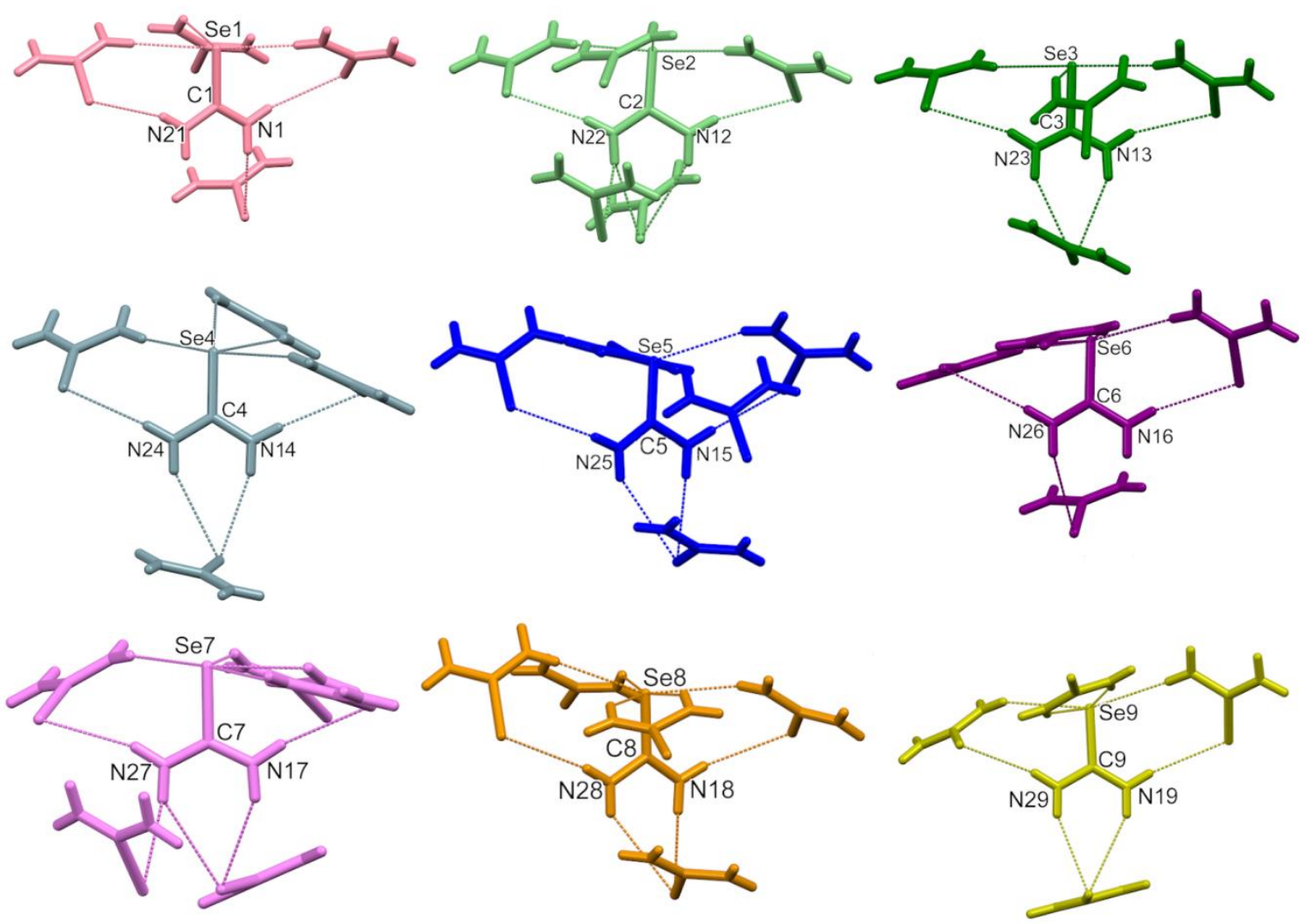

Figure S7 The components of Figure 6a shown for each of the central molecules viewed in the direction perpendicular to its average plane. The Se, $\mathrm{C}$ and $\mathrm{N}$ atoms are labelled for distinguishing the central molecules. 

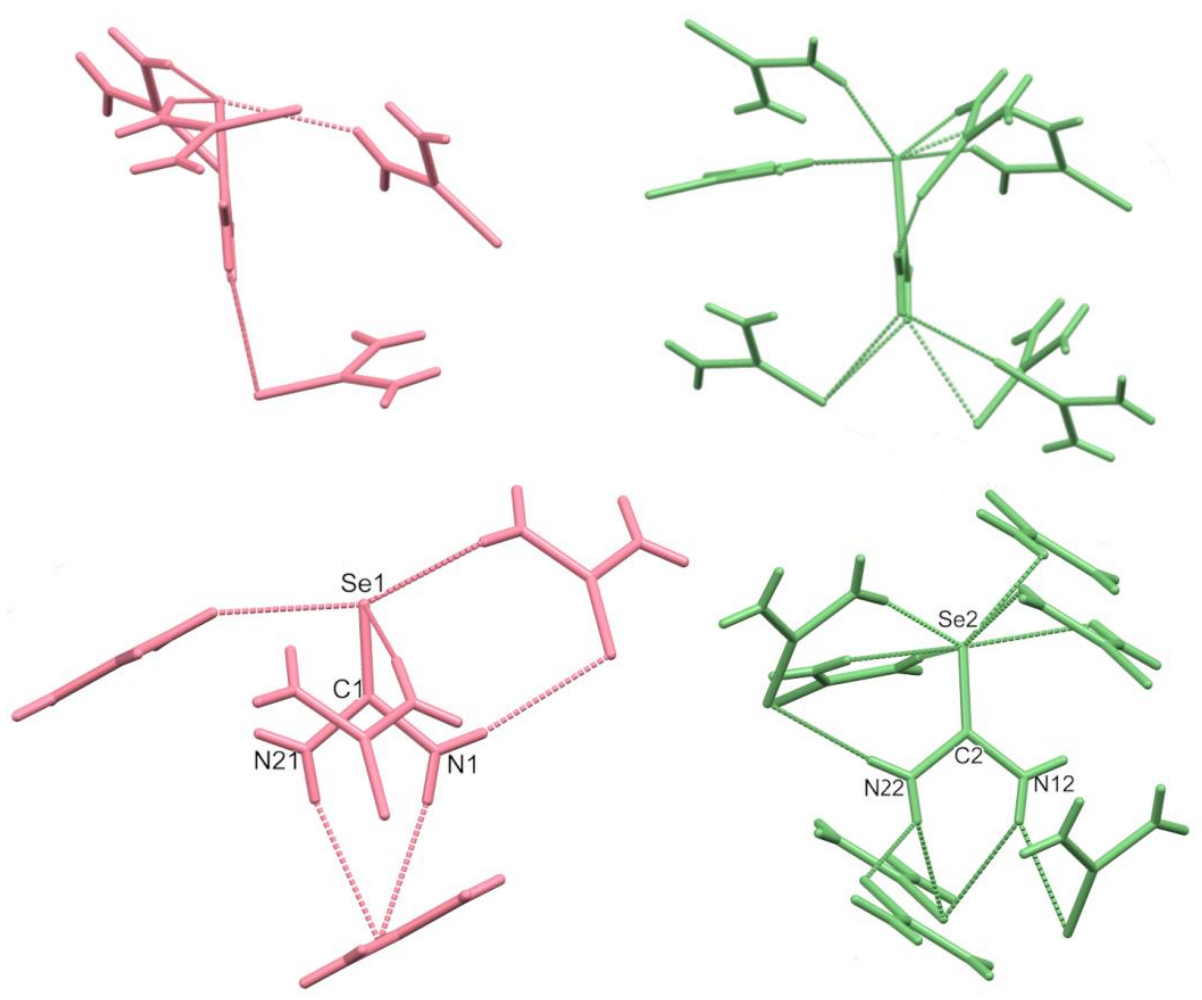

Figure S8 The components of Figure $6 \mathrm{~b}$ shown for each of the central molecules viewed in the direction perpendicular and parallel to its average plane. The Se, $\mathrm{C}$ and $\mathrm{N}$ atoms are labelled for distinguishing the central molecules. 

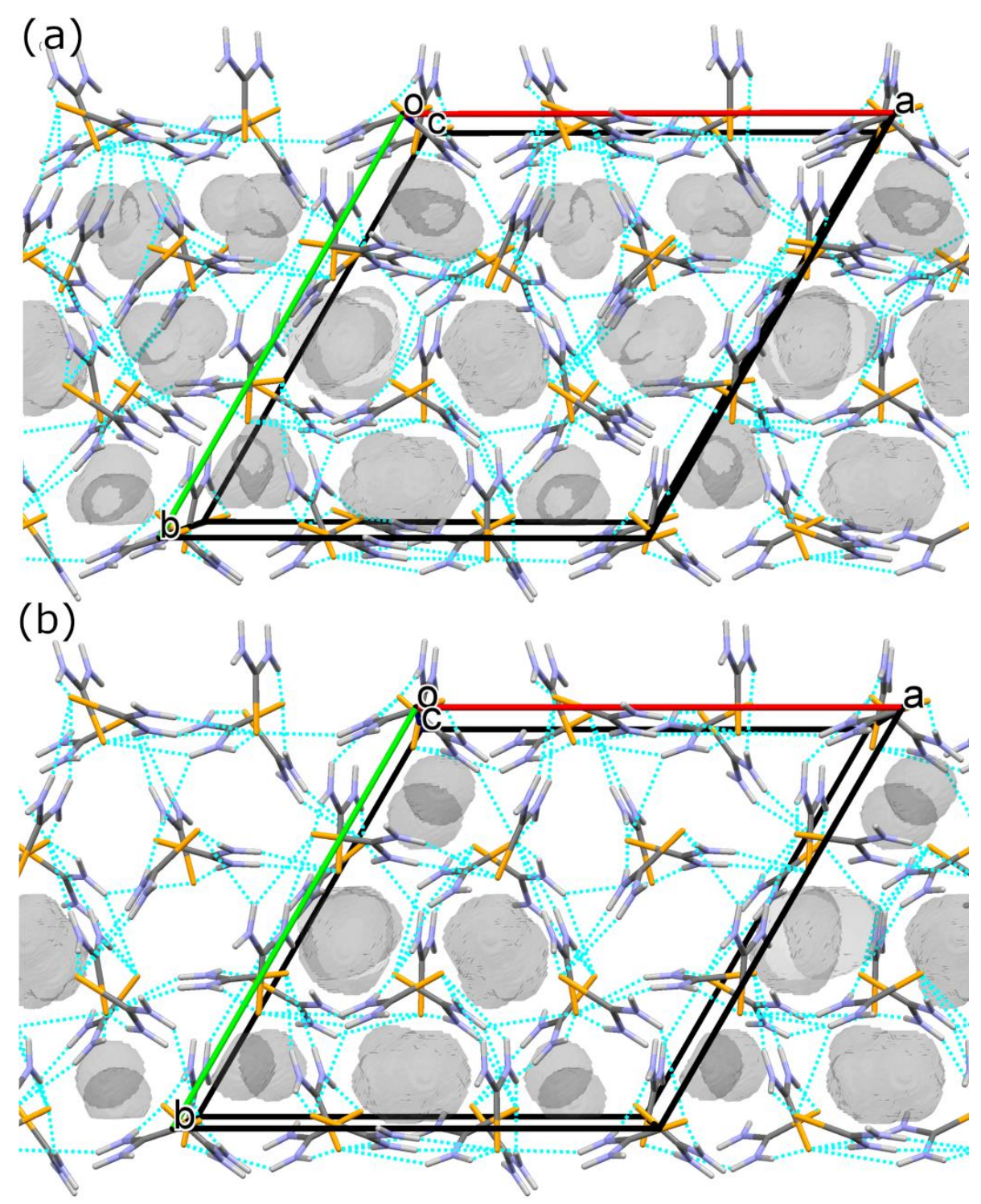

Figure S9 Crystal structure of $\mathrm{SeC}\left(\mathrm{NH}_{2}\right)_{2}$ phases $\alpha$ at: (a) $0.18 \mathrm{GPa}$ and (b) $0.20 \mathrm{GPa}$. Hydrogen bonds $\mathrm{NH} \cdots$ Se are indicated by blue dashed lines and the voids shown in yellow were calculated by Mercury (Macrae et al., 2020) for the probe radius of $1.0 \AA$ and grid step $0.1 \AA$. 


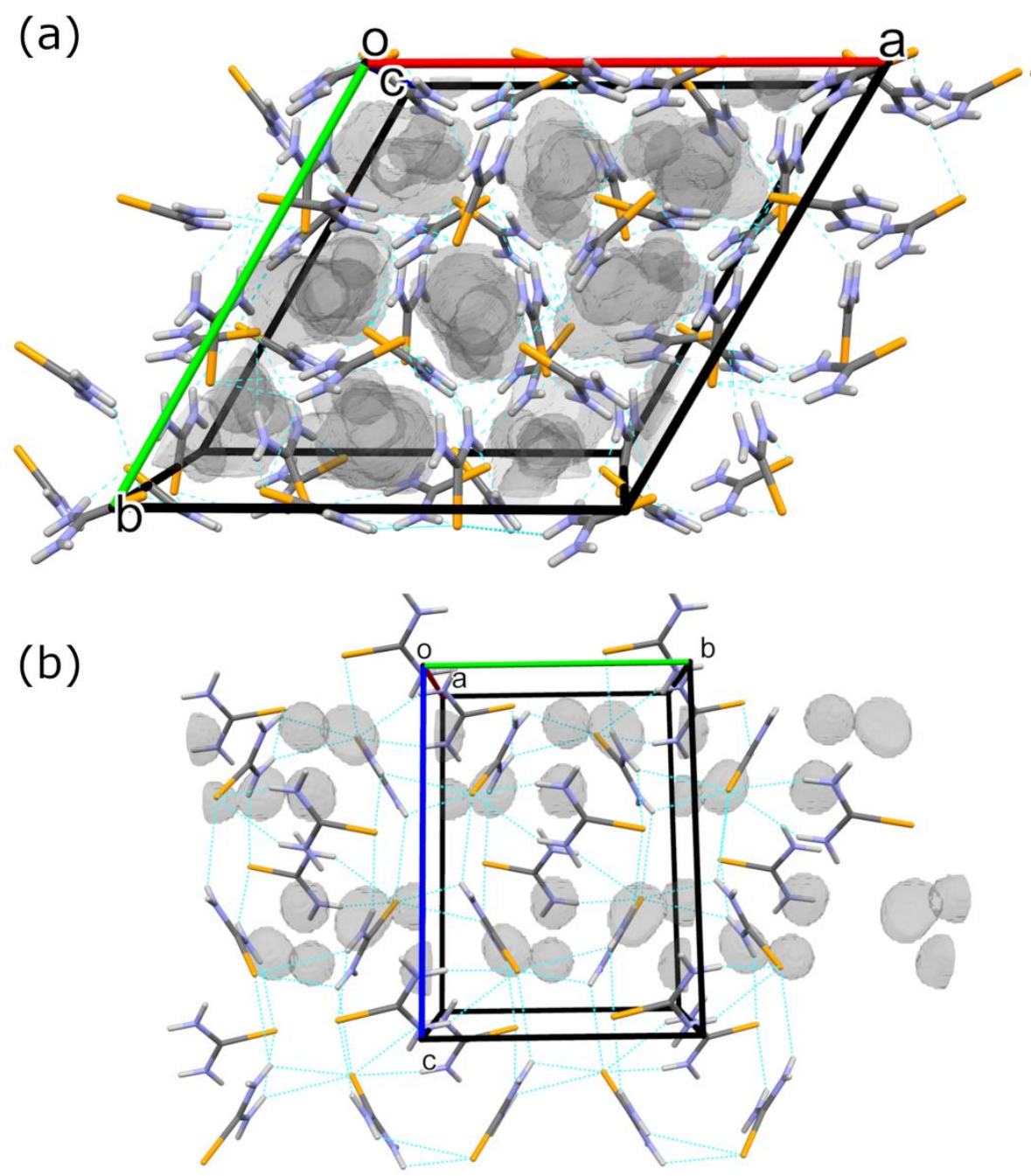

(c)

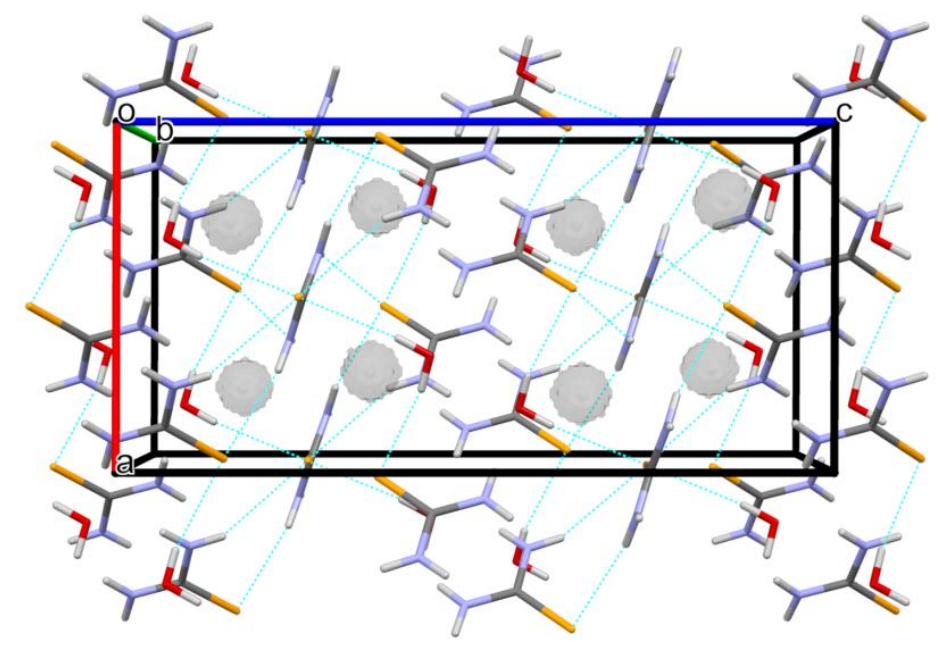

Figure S10Crystal structure of $\operatorname{SeC}\left(\mathrm{NH}_{2}\right)_{2}$ phases: (a) $\alpha$ at $0.1 \mathrm{MPa}$, (b) $\beta$ at $0.21 \mathrm{GPa}$; and (c) duotritohydrate at $0.52 \mathrm{GPa}$. Hydrogen bonds $\mathrm{NH} \cdots \mathrm{Se}$ and $\mathrm{OH} \cdots \mathrm{Se}$ in duotritohydrate are indicated by blue dashed lines and the voids shown in yellow were calculated by Mercury (Macrae et al., 2020) for the probe radius of $0.7 \AA$ and grid step $0.1 \AA$. 


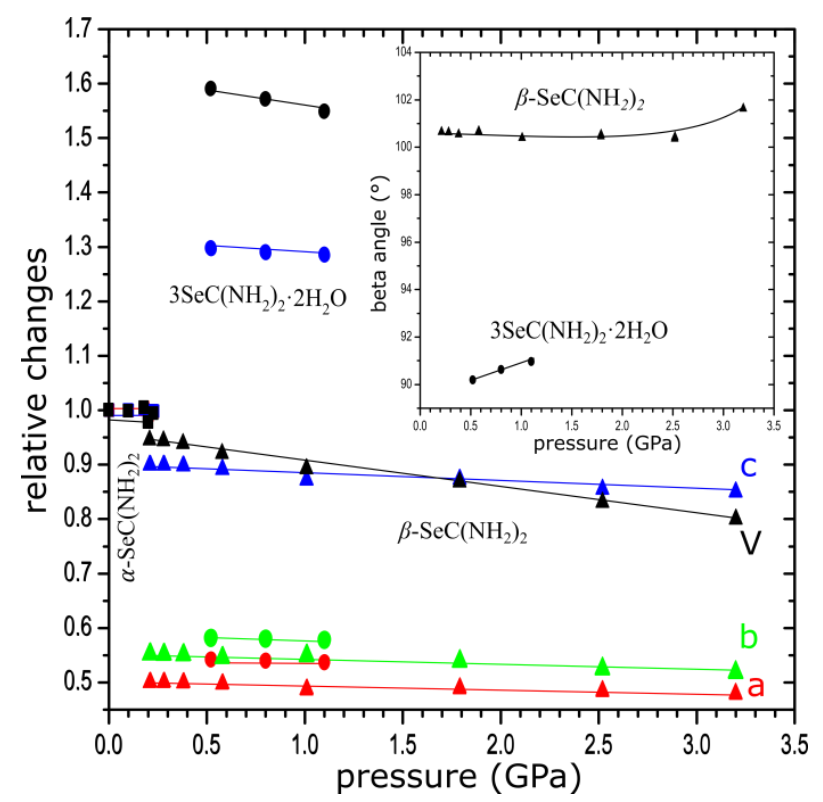

Figure S11 Compression of the unit-cell dimensions of $\mathrm{SeC}\left(\mathrm{NH}_{2}\right)_{2}$ polymorph $\alpha$ (square), polymorph $\beta$ (triangle) and $3 \mathrm{SeC}\left(\mathrm{NH}_{2}\right)_{2} \cdot 2 \mathrm{H}_{2} \mathrm{O}$ (circle) related to the $0.1 \mathrm{MPa} / 296 \mathrm{~K}$ values. The lines joining the points are for guiding the eye only. Estimated standard deviations are smaller than the plotted symbols.

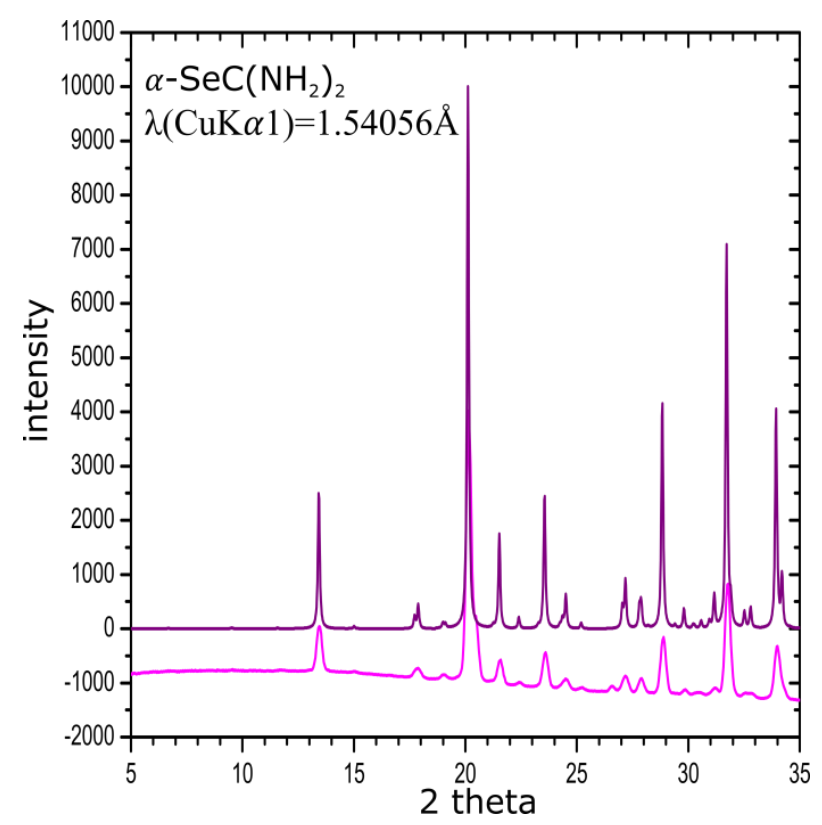

Figure S12Calculated (top) and measurement (bottom) X-ray powder diffraction pattern for $\alpha$ $\mathrm{SeC}\left(\mathrm{NH}_{2}\right)_{2}$ at $0.1 \mathrm{MPa}$ and $296 \mathrm{~K}$. 


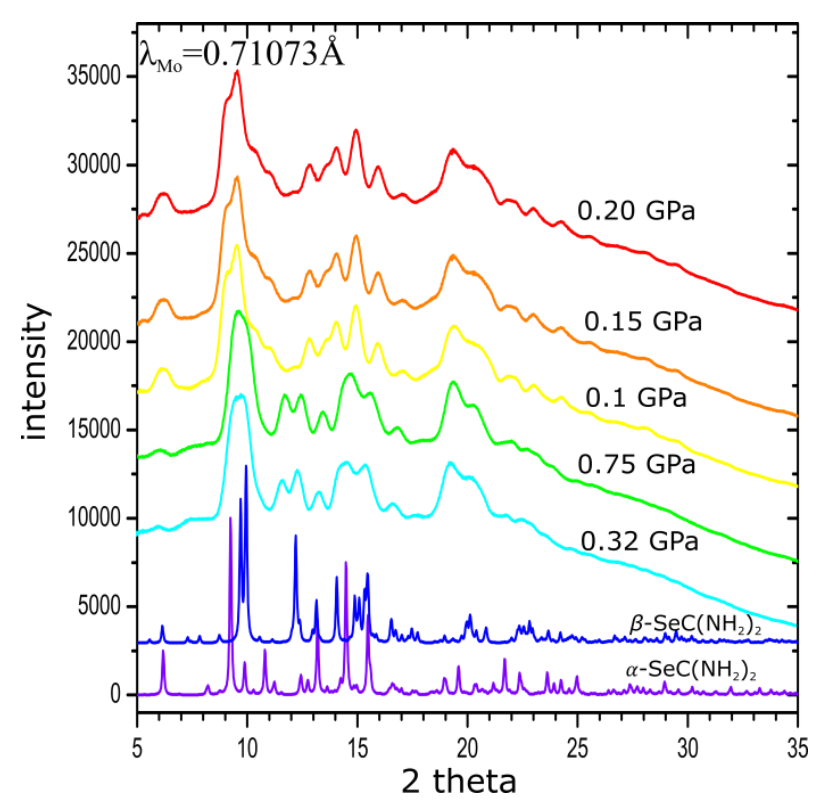

Figure S13 Calculated X-ray diffraction patterns for $\alpha-\mathrm{SeC}\left(\mathrm{NH}_{2}\right)_{2}$ and $\beta$ - $\mathrm{SeC}\left(\mathrm{NH}_{2}\right)_{2}$ (bottom), as well as the measured powder patterns for the sample compressed in glycerin in a DAC (top).

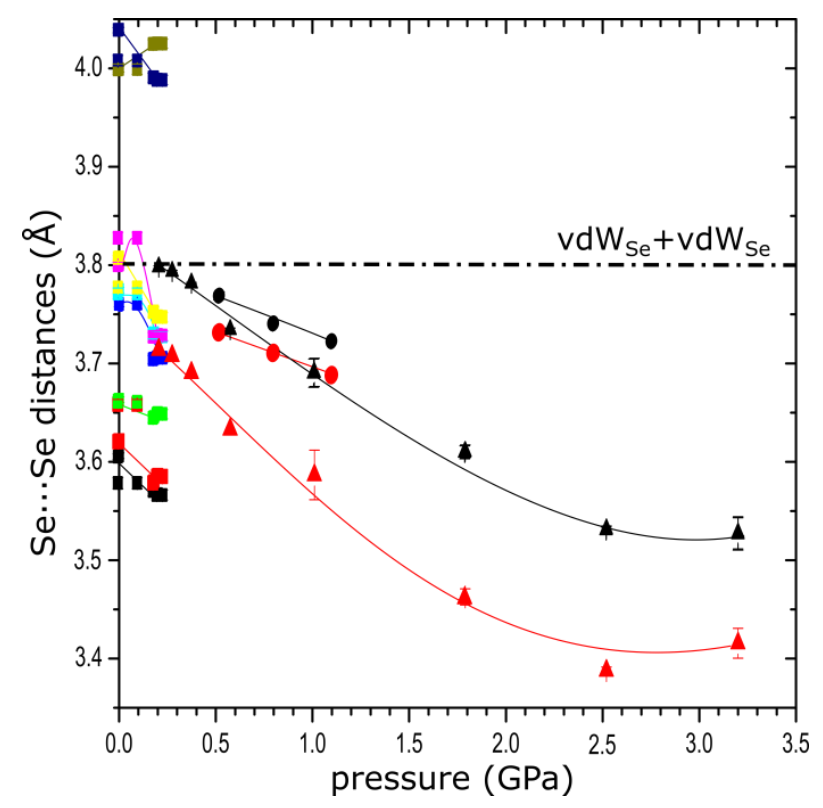

Figure S14 Distances Se $\cdots$ Se in the structure of $\mathrm{SeC}\left(\mathrm{NH}_{2}\right)_{2}$ phases $\alpha$ (square) and $\beta$ (triangle), as well as in $3 \mathrm{SeC}\left(\mathrm{NH}_{2}\right)_{2} \cdot 2 \mathrm{H}_{2} \mathrm{O}$ (circle), plotted as a function of pressure. 

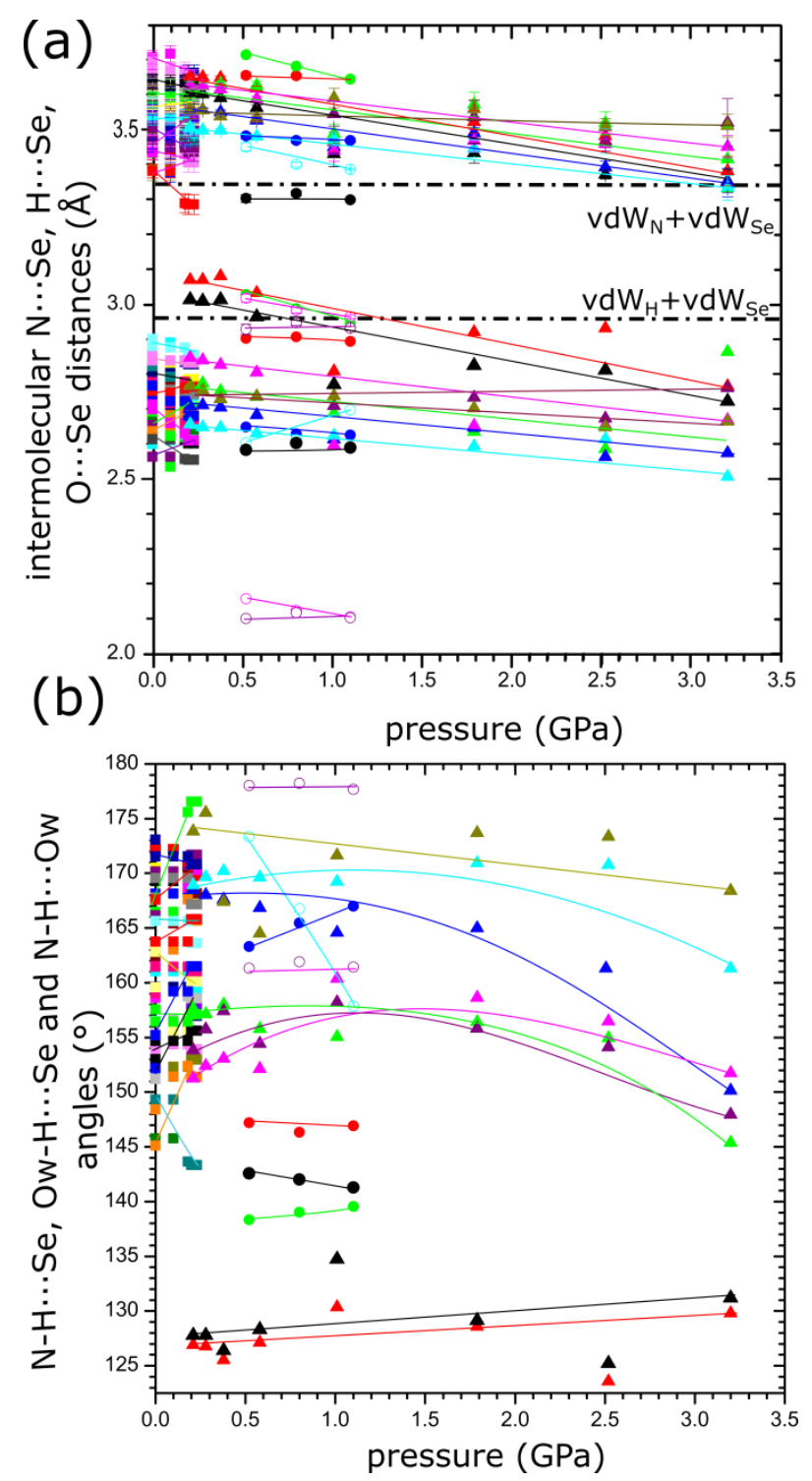

Figure S15 (a) Intermolecular distances and (b) angles Donor-H...Acceptor measured as a function of pressure in $\alpha-\mathrm{SeC}\left(\mathrm{NH}_{2}\right)_{2}, \beta-\mathrm{SeC}\left(\mathrm{NH}_{2}\right)_{2}$ and $3 \mathrm{SeC}\left(\mathrm{NH}_{2}\right)_{2} \cdot 2 \mathrm{H}_{2} \mathrm{O}$.
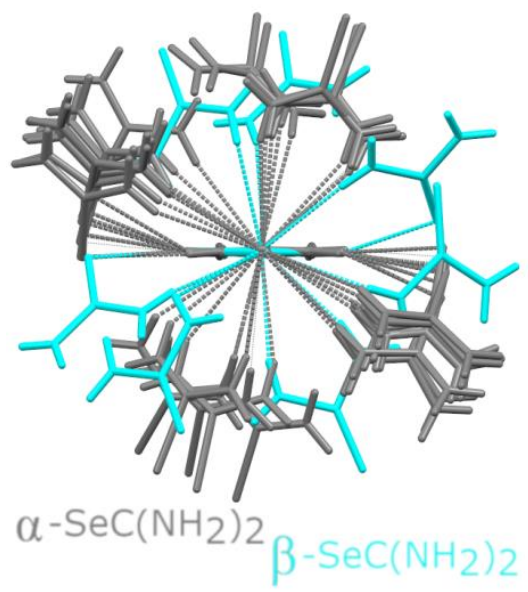

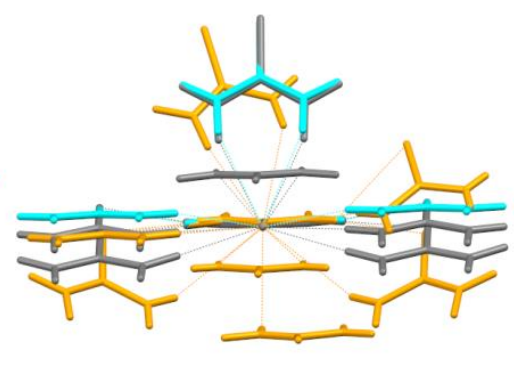

I SC $\left(\mathrm{NH}_{2}\right)_{2}$ $\checkmark \mathrm{SC}\left(\mathrm{NH}_{2}\right)_{2}$

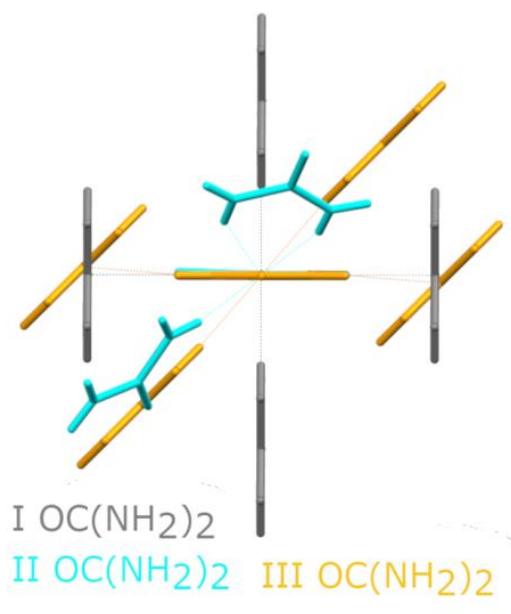




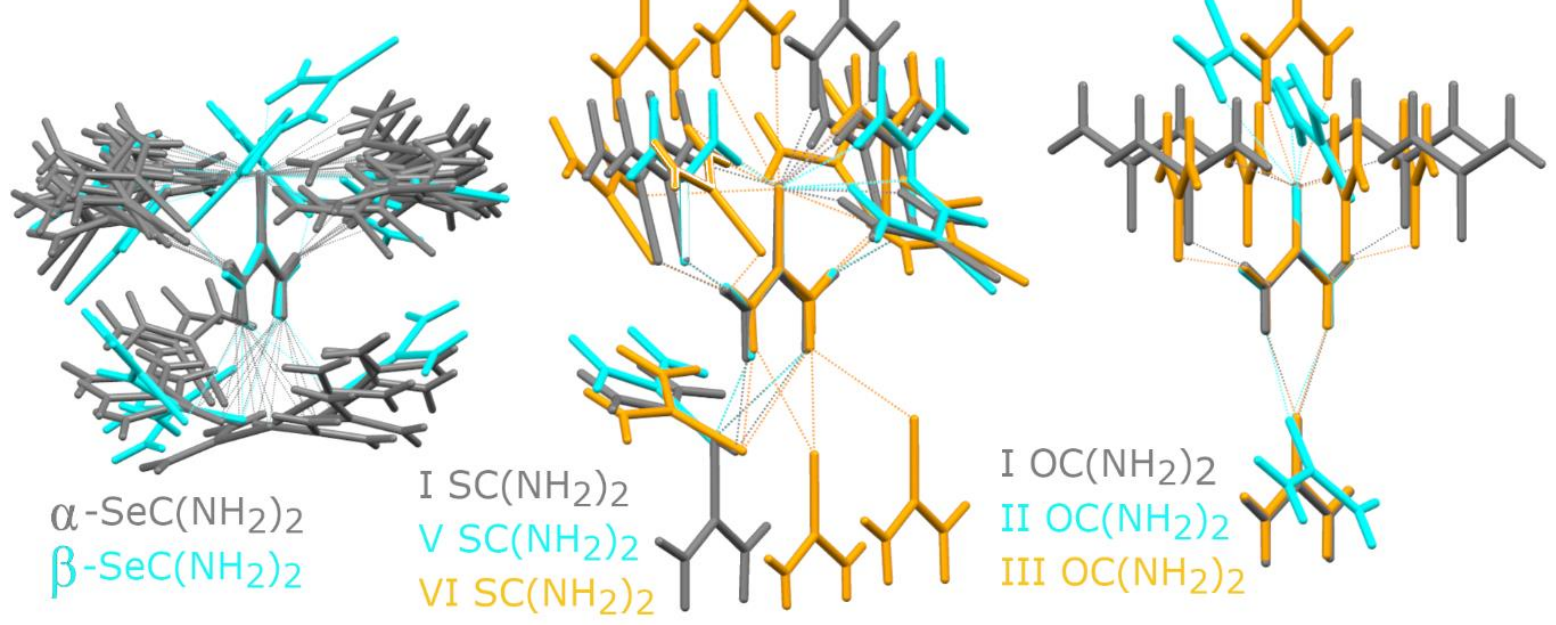

Figure S16 Exactly superimposed independent molecules and their $\mathrm{NH} \cdots \mathrm{X}(\mathrm{X}=\mathrm{Se}, \mathrm{O}, \mathrm{S})$ bonded neighbours in Selenourea (phases $\alpha$ and $\beta$ ); urea (phases I, III and IV) and thiourea (Phase I, V and VI). 